\title{
Impact of Irradiation on the Pharmacokinetics and Biotransformation of Tamoxifen
}

\author{
Yung-Yi Cheng ${ }^{1,2}$, Teresa Zheng ${ }^{1}$, Michael W. Chang ${ }^{1}$, Jeffrey W. Dalley ${ }^{3,4}$, \\ Yu-Jen Chen ${ }^{5,6,7,8^{*}}$, Tung-Hu Tsai ${ }^{2,3^{*}}$ and Chen-Hsi Hsieh ${ }^{2,9,10^{*}}$
}

${ }^{1}$ Division of Pharmacoengineering and Molecular Pharmaceutics, Eshelman School of Pharmacy, the University of North Carolina at Chapel Hill, Chapel Hill, NC, United States, ${ }^{2}$ Institute of Traditional Medicine, National Yang Ming Chiao Tung University, Taipei, Taiwan, ${ }^{3}$ Department of Psychology, University of Cambridge, Cambridge, United Kingdom, ${ }^{4}$ Department of Psychiatry, University of Cambridge, Cambridge, United Kingdom, ${ }^{5}$ Department of Radiation Oncology, MacKay Memorial Hospital, Taipei, Taiwan, ${ }^{6}$ Department of Artificial Intelligence and Medical Application, MacKay Junior College of Medicine, Nursing and Management, Taipei, Taiwan, ${ }^{7}$ Department of Medical Research, China Medical University Hospital, Taichung, Taiwan, ${ }^{8}$ Department of Medical Research, MacKay Memorial Hospital, New Taipei City, Taiwan, ${ }^{9}$ Faculty of Medicine, School of Medicine, National Yang Ming Chiao Tung University, Taipei, Taiwan, ${ }^{10}$ Division of Radiation Oncology, Department of Radiology, Far Eastern Memorial Hospital, New Taipei City, Taiwan

\section{OPEN ACCESS}

Edited by:

Shih-Kai Hung,

Dalin Tzu Chi Hospital, Taiwan

Reviewed by:

Yu-Tse Wu,

Kaohsiung Medical University, Taiwan

Yu-Chuen Huang,

China Medical University, Taiwan

${ }^{*}$ Correspondence:

Yu-Jen Chen

chenmdphd@gmail.com

Tung-Hu Tsai

thtsai@nycu.edu.tw

Chen-Hsi Hsieh

chenicab@gmail.com;

chenci28@ym.edu.tw

Specialty section: This article was submitted to

Radiation Oncology,

a section of the journal

Frontiers in Oncology

Received: 10 December 2021 Accepted: 24 January 2022

Published: 17 February 2022

Citation:

Cheng Y-Y, Zheng T, Chang MW,

Dalley JW, Chen Y-J, Tsai T-H and Hsieh C-H (2022) Impact of Irradiation

on the Pharmacokinetics and

Biotransformation of Tamoxifen.

Front. Oncol. 12:833108.

doi: 10.3389/fonc.2022.833108
Background: The optimal procedure for combining radiotherapy (RT) with tamoxifen treatment is controversial as RT may alter the pharmacokinetics and biotransformation of tamoxifen. The present study investigated this potential interaction by assessing the pharmacokinetics of tamoxifen during concurrent and sequential RT.

Method: Plasma tamoxifen concentration was measured in rats with or without RT 2.0 Gy $\left(R T_{2.0 G y}\right)$ or 0.5 Gy $\left(R T_{0.5 G y}\right)$ with ultra-high-performance liquid chromatography-tandem mass spectrometry after tamoxifen administration (10 mg/ $\mathrm{kg}$, p.o., $n=6$ ). Tamoxifen was either administered $1 \mathrm{~h}$ after RT (concurrent condition) or $24 \mathrm{~h}$ after RT (sequential condition).

Results: Pharmacokinetic data analysis demonstrated that the area under the curve (AUC) and half-life of tamoxifen were $2,004 \pm 241 \mathrm{~h} \mathrm{ng} / \mathrm{ml}$ and $6.23 \pm 1.21 \mathrm{~h}$, respectively, after tamoxifen administration (10 mg/kg, p.o.). The respective conversion rate of 4hydroxytamoxifen, $\mathrm{N}$-desmethytamoxifen, and endoxifen for tamoxifen metabolism was $20 \%, 16 \%$, and $5 \%$. The AUC value of tamoxifen in the RT 0.5 Gy group was 1.5 - to 1.7 -fold higher than in the sham and $\mathrm{RT}_{2.0 \mathrm{~Gy}}$ groups. The relative bioavailability of tamoxifen at concurrent $\mathrm{RT}_{0.5 \mathrm{~Gy}}$ and $\mathrm{RT}_{2.0 \mathrm{~Gy}}$ groups ranged from $127 \%$ to $202 \%$ and from $71 \%$ to $152 \%$, respectively. The magnitude of endoxifen, which converted from 4hydroxytamoxifen and $\mathrm{N}$-desmethyltamoxifen, increased 3 - to 5-fold in the concurrent RT groups. By contrast, the AUC of tamoxifen decreased by roughly $24 \%$ in the sequential $\mathrm{RT}_{2.0 \mathrm{~Gy}}$ group. The conversion ratio of endoxifen was four times higher than that in the sequential $\mathrm{RT}_{2.0 \mathrm{~Gy}}$ group compared with rats not exposed to $\mathrm{RT}$.

Conclusion: The current study provides advanced pharmacokinetic data to confirm the interaction between RT and hormone therapy. Our findings indicate that RT facilitates the metabolism of tamoxifen to active metabolites and thus imply that combination RT-tamoxifen has potential benefits for the treatment of hormone-dependent breast cancer.

Keywords: breast cancer, radiotherapy, tamoxifen, pharmacokinetics, tandem mass spectrometry 


\section{INTRODUCTION}

Breast cancer is one of the top 10 diagnosed cancers for women, accounting for $11.7 \%$ of total new cases (1). Current treatment strategies for breast cancer include surgery, chemotherapy, endocrine therapy, radiotherapy (RT), immunotherapy, and combinations of these options. The National Surgical Adjuvant Breast and Bowel Project (NSABP) B-06 study demonstrated that the rate of local recurrence after a 20 -year follow-up was $39 \%$ for patients treated with lumpectomy alone or $14 \%$ for patients treated with both lumpectomy and RT (2). The 10-year overall survival of patients was increased for patients who underwent RT, as reported in the MA.20 trial and the European Organization for Research and Treatment of Cancer (EORTC) trial $(3,4)$.

Four Phase III randomized trials have prospectively evaluated tamoxifen with placebo for breast cancer risk reduction (5-8). The relative risk was reduced by $34 \%-50 \%$ for patients who received 5year adjuvant tamoxifen therapy $(5,7,9)$. Tamoxifen has been prescribed as monotherapy or combined with RT for many years. Ellis and coworkers observed that estrogen-positive breast cells pretreated with tamoxifen had a greater apoptosis index and implies the radiosensitivity of tamoxifen (10). Correspondingly, the NSABP-B14 trial suggested that tamoxifen and RT may have a synergistic interaction since patients receiving both therapies experienced better outcomes in terms of local control (9). Nevertheless, breast cancer patients treated with tamoxifen and RT were 2- to 3-fold more likely to develop radiation-induced pulmonary fibrosis $(11,12)$. Of note, there were no significant differences in the recurrence, disease-free survival, or overall survival of breast cancer patients treated with tamoxifen concurrent or sequential with RT (13).

$\mathrm{RT}$ is associated with reactive oxygen species (ROS) production (14). Moreover, the primary sources of ROS in the liver are the mitochondria and cytochrome P450 enzyme systems and derive from Kupffer and inflammatory cells (15). Additionally, drug pharmacokinetics influenced by RT has previously been revealed (16, 17). Breast cancer patients treated by advanced RT could experience off-target exposure to surrounding organs such as the lung and heart, which might cause unpredictable effects $(18,19)$. Recently, RT significantly impacted the hepatic microsomal cytochrome P450 3A4 (CYP3A4) activity and P-glycoprotein (P-gp) activity $(16,17)$. This was likely caused by an unintended interaction between RT and tamoxifen. However, few preclinical studies have specifically investigated the interaction between tamoxifen and RT and how this affects the pharmacokinetics and metabolism of tamoxifen. The present study focused on the RT interaction with tamoxifen and its metabolites using two RT sequences.

\section{MATERIALS AND METHODS}

\section{Reagents and Chemicals}

Tamoxifen citrate salt (>98\%) was obtained from TCI chemicals (TCI, Portland, Oregon, USA). 4-hydroxytamoxifen (>98\%), $N$-desmethyltamoxifen hydrochloride (>98\%), E/Z endoxifen hydrochloride (>98\%) and heparin were obtained from Sigma-
Aldrich Chemical Co. (St. Louis, MO, USA). 5-methylflavone (internal standard) was purchased from Tokyo Chemical Industry. LC-MS grade organic solvents, including methanol, acetonitrile, formic acid, and ammonium bicarbonate, were acquired from Merck Co. (Darmstadt, Germany). Purified deionized water was produced using a Milli-Q system (Millipore, Milford, MA, USA).

\section{Animals}

Female Sprague-Dawley rats $(200 \pm 20$ g) were obtained from the Laboratory Animal Centre at National Yang Ming Chiao Tung University (Taipei, Taiwan). Food (Laboratory Rodent Diet 5001, PMI Nutrition International LLC, MO, USA) and water were supplied ad libitum. All experimental procedures involving surgery were reviewed and approved by the Institutional Animal Care and Use Committee of National Yang Ming Chiao Tung University (IUCAC no.1051204). For the pharmacokinetic studies, catheterization of the carotid artery was used in unrestrained conscious rats (16). Rats were anesthetized with pentobarbital sodium (50 mg/kg, IP injection, Taoyuan, Taiwan) and implanted with PE-50 polyethylene (I.D. $0.58 \mathrm{~mm} \times$ O.D. $0.965 \mathrm{~mm}, \mathrm{MD}$, USA) tubing in the left carotid artery. The exteriorized catheter was secured in the dorsal neck area and capped with a stopper. Normal heparinized saline (heparin $500 \mathrm{IU} / \mathrm{ml}$ in normal saline) was used to maintain the patency of the tubing. Rats were allowed a minimum of $24 \mathrm{~h}$ to recover prior to drug administration. Blood samples (150 $\mu \mathrm{l})$ were collected from the jugular vein at $0.25,0.5,1,2,4,6,8,10$, $12,16,18,20$, and $24 \mathrm{~h}$ after each drug administration. Plasma was separated by centrifugation at $6000 \times \mathrm{g}$ for $10 \mathrm{~min}$ at $4^{\circ} \mathrm{C}$ and stored at $-20^{\circ} \mathrm{C}$ prior to further analysis. After finishing the experiment, an overdose of $\mathrm{CO}_{2}$ was used to euthanize animals.

The dose of tamoxifen used daily in humans is typically 20 to $40 \mathrm{mg}(20,21)$. Based on the human-animal dose conversion formula: human equivalent dose (HED, $\mathrm{mg} / \mathrm{kg}$ ) = animal dose $(\mathrm{mg} / \mathrm{kg}) \times$ animal $\mathrm{km}$ (body weight $(\mathrm{kg})$ divided by body surface area (BSA) $\left(\mathrm{m}^{2}\right) /$ human $\mathrm{km}(22)$, the range of tamoxifen would then be orally $2.0-4.1 \mathrm{mg} / \mathrm{kg}$ in rats. Plasma drug levels are probably below the lowest detectable concentration of quantification, given that the bioavailability of tamoxifen was approximately 20\% (23). The tamoxifen dosage orally administered in the present study is consistent with our previous work (24). Experimental animals were randomized into five groups: group 1 (sham group): tamoxifen $10 \mathrm{mg} / \mathrm{kg}$, p.o. plus $0 \mathrm{~Gy}$; two concurrent tamoxifen with single-fraction irradiation; group 2: tamoxifen $10 \mathrm{mg} / \mathrm{kg}$, p.o. plus RT $0.5 \mathrm{~Gy}\left(\mathrm{RT}_{0.5 \mathrm{~Gy}}\right)$; group 3: tamoxifen $10 \mathrm{mg} / \mathrm{kg}$, p.o. plus RT $2.0 \mathrm{~Gy}\left(\mathrm{RT}_{2 \mathrm{~Gy}}\right)$; sequential tamoxifen with single-fraction irradiation; group 4: tamoxifen $10 \mathrm{mg} / \mathrm{kg}$, p.o. plus RT $0.5 \mathrm{~Gy}\left(\mathrm{RT}_{0.5 \mathrm{~Gy}}\right)$, and group 5: tamoxifen $10 \mathrm{mg} / \mathrm{kg}$, p.o. plus RT $2.0 \mathrm{~Gy}\left(\mathrm{RT}_{2 \mathrm{~Gy}}\right)$. Rats received tamoxifen 1 hour after RT represented as the concurrent regimen. Receiving tamoxifen $24 \mathrm{~h}$ after RT was deemed the sequential regimen. Data were obtained from 6 rats in each group.

\section{Irradiation Technique}

Rats were anesthetized with pentobarbital sodium (50 mg/kg, i.p.) and immobilized on a board to undergo computed tomography to simulate the breast field. The cranial margin was set at the clavicle 
head and sternum junction with a $2.5 \times 4 \mathrm{~cm}$ irradiation field. Conventional RT technique was employed to deliver the irradiation dose via the anterior portal with a depth of $0.5 \mathrm{~cm}$ to the left side chest wall of rats. In the clinical cases treated with different irradiation techniques, more than $50 \%$ of the normal liver was exposed to 0.5 Gy (off-target dose) during daily 2-Gy radiation treatments (25). Reviewing several related studies, currently, there is no direct comparison of allometric scaling using chest wall irradiation between humans and rodents. However, the respective lethal dose (LD50) of total-body irradiation for human and rat is 4 and 6.75 Gy (26). The LD50 is defined as the dose that causes a mortality rate of $50 \%$ in an experimental group within a specified period of time. Our previous report demonstrated that irradiation of 2 Gy to the rats is safe and workable to stimulate the relevant dose for daily treatment of the human torso. RT 0.5 Gy represented a dose deposited in the off-target area in clinical practice (25). According to the above data, the irradiation doses of 0.5 and 2 Gy for rats simulating the relevant dose range for daily treatment of the human torso were utilized in the current study.

\section{Sample Preparation}

The primary stock solution of tamoxifen, 4-hydroxytamoxifen, $\mathrm{N}$ desmethyltamoxifen, and endoxifen was $1 \mathrm{mg} / \mathrm{ml}$ in methanol and was stored at $-20^{\circ} \mathrm{C}$ until analysis. The working solutions of calibration curves for quantification analysis were diluted using acetonitrile. For calibration curves, the drug-free plasma $(50 \mu \mathrm{l})$ and $10-\mu \mathrm{l}$ working solution were mixed with $140 \mu \mathrm{l}$ of internal standard solution (acetonitrile containing $50 \mathrm{ng} / \mathrm{ml}$ internal standard, ACNIS solution). The mixed samples were then vortexed for $5 \mathrm{~min}$ and centrifuged for $10 \mathrm{~min}$ at the speed of 15,000 r.p.m. The collected supernatant was filtered through a $0.22-\mu \mathrm{m}$ filter before LCMS analysis. Plasma samples were extracted using $150 \mu \mathrm{l}$ of ACN-IS solution. All prepared samples were kept at $10^{\circ} \mathrm{C}$ in the autosampler throughout the analysis.

\section{UPLC-MS/MS for Quantification Analysis}

Ultra-high-performance liquid chromatography-tandem triplequadrupole mass spectrometry (Waters Acquity B.S.M.) equipped with an electrospray ionization device (Waters Xevo TQ MS, Milford, MA, USA) was used in this study to simultaneously determine tamoxifen and its metabolites, including 4hydroxytamoxifen, $\mathrm{N}$-desmethyltamoxifen, and endoxifen. Chromatographic separation was performed by the optimized elution, consisting of $10 \mathrm{mM}$ ammonium bicarbonate and methanol (15/85) with a flow rate of $0.2 \mathrm{ml} / \mathrm{min}$, and using a C18 column $(\mathrm{C} 18,100 \times 2.1 \mathrm{~mm}, 2.7 \mu \mathrm{m}$; Dikama, B.S.M., maintained at $40^{\circ} \mathrm{C}$ ) to accomplish the analysis. The sample injection volume was $5 \mu \mathrm{l}$. The MS/MS condition and analyte transition have previously been described (24). The coefficient correlation $\left(r^{2}\right)$ as the linearity standard was at least 0.995 . The relative standard deviation (RSD, $\%$ ) was precision calculated by RSD (\%) = (standard deviation/ observed concentration) $\times 100 \%$. The closeness of a measured value to the mean value for the actual value was accuracy (bias, \%), which was calculated as follows: accuracy $(\%)=[$ (the nominal concentration - the observed concentration)/the nominal concentration] $\times 100 \%$. The acceptable value of precision and accuracy was within $\pm 15 \%$, except the lower limit of quantification (LLOQ, defined as a signal-to-noise ratio of less than 10). The precision and accuracy of the LLOQ were always lower than $\pm 20 \%$.

\section{Pharmacokinetic Analysis and Statistical Analysis}

PK parameters, including the area under the concentration-time curve (AUC), terminal elimination phase half-life $\left(t_{1 / 2}\right)$, maximum observed plasma concentration $\left(\mathrm{C}_{\max }\right)$, and time of maximum concentration observed $\left(\mathrm{T}_{\max }\right)$, were calculated using the PK calculation software WinNonlin Standard Edition, Version 1.1 (Scientific Consulting, Apex, NC, USA). Relative bioavailability $(\mathrm{RB} \%)=\left(\mathrm{AUC}_{\text {irradiated }} / \mathrm{AUC}_{\text {control }}\right) \times 100$. The metabolic conversion of tamoxifen was calculated using the following formula: Metabolite conversion $(\mathrm{MC} \%)=\left(\mathrm{AUC}_{\text {metabolite }} /\right.$ $\left.\mathrm{AUC}_{\text {parent }}\right) \times 100$. The results were presented as means \pm standard deviations. Differences in actuarial outcomes between the groups were calculated by one-way analysis of variance (ANOVA) and post-hoc Student-Newman-Keuls tests using the SigmaPlot program (Systat Software Inc., version 6.0). A p-value of $<0.05$ was considered statistically significant.

\section{RESULTS}

\section{Validation of the UPLC-MS/MS Method}

To optimize peak shape and resolution, the composition of the mobile phase and analytical method was carefully adjusted. The robust UPLC-MS/MS-based analytic method to determine the blood concentration of tamoxifen and its major metabolites was established and validated. The retention time of tamoxifen, 4-hydroxytamoxifen, $\mathrm{N}$-desmethyltamoxifen, and endoxifen individually were $4.4,2.3,3.2$, and $1.9 \mathrm{~min}$, respectively. The linear concentration ranges with acceptable coefficient correlation $\left(r^{2}>0.995\right)$ of tamoxifen, 4-hydroxytamoxifen, $\mathrm{N}$-desmethyltamoxifen, and endoxifen were $1-1,000 \mathrm{ng} / \mathrm{ml}$. The accuracy and precision data of the relevant concentration ranges enabled effective quantification. The accuracy and precision of intraday ranged from 0.15 to 15.20 and from -13.52 to 12.78 , respectively. The accuracy and precision of inter-day were within 0.16-13.12 and -13.48-7.04, respectively. The accuracy and precision of the relevant concentration ranges were thus acceptable for quantification.

\section{Pharmacokinetics and Biotransformation of Tamoxifen in Plasma}

The profile of the plasma concentration-time curves of tamoxifen and its metabolites after female rats single orally administered tamoxifen is illustrated in Figure 1. Tamoxifen reached the $\mathrm{C}_{\max }(143.9 \pm 25.2 \mathrm{ng} /$ $\mathrm{ml}$ ) after approximately $5.5-8.6 \mathrm{~h}\left(\mathrm{~T}_{\max }\right)$ after orally receiving tamoxifen. A non-compartmental model was used to analyze the pharmacokinetic data. The pharmacokinetic parameters of tamoxifen and its metabolites are summarized in Table 1. The AUC of tamoxifen, 4-hydroxytamoxifen, $\mathrm{N}$-desmethyltamoxifen, and endoxifen was $2,004 \pm 241,385.7 \pm 149.8,283.7 \pm 61.8$, and $92.7 \pm 44.2 \mathrm{~h} \mathrm{ng} / \mathrm{ml}$, respectively. The half-lives of tamoxifen, 4-hydroxytamoxifen, 


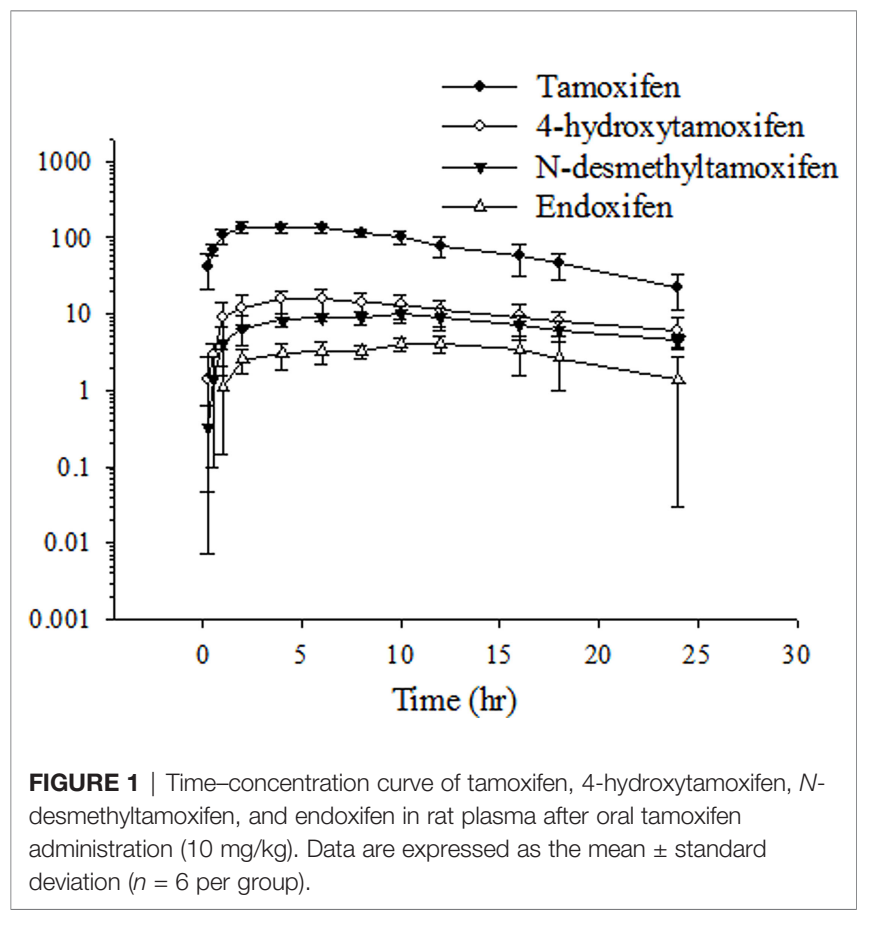

$N$-desmethyltamoxifen, and endoxifen was $6.23 \pm 1.21,13.6 \pm 6.4,16.4$ \pm 10.4 , and $7.48 \pm 2.57 \mathrm{~h}$, respectively. Additionally, the biotransformation of tamoxifen was evaluated by metabolic conversion, calculated by the metabolite-to-tamoxifen AUC ratio for 4-hydroxytamoxifen, $N$-desmethyltamoxifen, and endoxifen. Based on the formula of metabolic conversion, the metabolic conversion of 4hydroxytamoxifen, $N$-desmethyltamoxifen, and endoxifen was $19.6 \pm$ $8.1 \%, 16.1 \pm 2.4 \%$, and $4.73 \pm 2.27 \%$, respectively. The metabolic conversion of 4-hydroxytamoxifen ranged from approximately $20 \%$ and was compatible with previous studies (24). However, the related studies rarely investigated the metabolic conversion of $\mathrm{N}$ desmethyltamoxifen and endoxifen. Our present study showed the magnitude of $5 \%$ conversion of endoxifen for tamoxifen metabolism, and 4-hydroxytamoxifen and $N$-desmethyltamoxifen had a similar ratio of conversion.

\section{Pharmacokinetics and Biotransformation of Tamoxifen During the Concurrent Regimen}

Rats received tamoxifen $1 \mathrm{~h}$ after radiation (concurrent regimen) to determine effects on pharmacokinetic variables in the sham, $\mathrm{RT}_{0.5 \mathrm{~Gy}}$, and $\mathrm{RT}_{2.0 \mathrm{~Gy}}$ groups. Plasma concentration curves are illustrated in Figures 2 and 3. The relevant non-compartmental parameters are listed in Table 1. At the $\mathrm{RT}_{0.5 \mathrm{~Gy}}$ group, the $\mathrm{C}_{\max }$

TABLE 1 | The parameters of pharmacokinetic in tamoxifen, 4-hydroxytamoxifen, $\mathrm{N}$-desmethyltamoxifen, and endoxifen for rats treated with tamoxifen (10 mg/kg, p.o.) concurrent or sequential with irradiation $0.5 \mathrm{~Gy}\left(\mathrm{RT}_{0.5 \mathrm{~Gy}}\right)$ and $2 \mathrm{~Gy}\left(\mathrm{RT}_{2.0 \mathrm{~Gy}}\right)$.

\begin{tabular}{|c|c|c|c|c|c|}
\hline \multirow{3}{*}{ Pharmacokinetic parameters } & \multicolumn{5}{|c|}{ Tamoxifen (10 mg/kg, p.o.) } \\
\hline & \multirow{2}{*}{$\begin{array}{l}\text { Sham } \\
\text { o Gy }\end{array}$} & \multicolumn{2}{|c|}{ Concurrent } & \multicolumn{2}{|c|}{ Sequential } \\
\hline & & $0.5 \mathrm{~Gy}$ & $2.0 \mathrm{~Gy}$ & $0.5 \mathrm{~Gy}$ & $2.0 \mathrm{~Gy}$ \\
\hline \multicolumn{6}{|l|}{ Tamoxifen } \\
\hline AUC (h ng/ml) & $2,004 \pm 241$ & $3,468 \pm 722$ & $2,089 \pm 582$ & $2,021 \pm 299$ & $1,530 \pm 167^{\star \# \mathbb{P}}$ \\
\hline $\mathrm{t}_{1 / 2}(\mathrm{~h})$ & $6.23 \pm 1.21$ & $5.54 \pm 1.10$ & $7.14 \pm 1.85$ & $5.85 \pm 1.30$ & $5.28 \pm 1.66$ \\
\hline $\mathrm{T}_{\max }(\mathrm{h})$ & $4.67 \pm 1.63$ & $5.00 \pm 1.09$ & $8.00 \pm 2.83$ & $4.17 \pm 2.23$ & $5.67 \pm 2.66$ \\
\hline $\mathrm{C}_{\max }(\mathrm{ng} / \mathrm{ml})$ & $143.9 \pm 25.2$ & $270.4 \pm 66.3$ & $135.8 \pm 38.9$ & $156.6 \pm 43.7$ & $108.0 \pm 13.9$ \\
\hline Relative bioavailability (\%) & 100 & $173.0 \pm 36.1$ & $104.3 \pm 29.1$ & $107.3 \pm 21.7$ & $76.4 \pm 8.3^{\star \# \mathbb{R}}$ \\
\hline \multicolumn{6}{|l|}{ 4-Hydroxytamoxifen } \\
\hline AUC (h ng/ml) & $385.3 \pm 149.8$ & $705.1 \pm 103.0$ & $526.9 \pm 138.7$ & $524.0 \pm 130.0$ & $331.2 \pm 37.8$ \\
\hline$t_{1 / 2}(h)$ & $13.6 \pm 6.4$ & $6.59 \pm 1.88$ & $7.59 \pm 2.07$ & $7.05 \pm 3.09$ & $5.79 \pm 5.10$ \\
\hline $\mathrm{T}_{\max }(\mathrm{h})$ & $5.0 \pm 1.1$ & $5.33 \pm 1.03$ & $9.33 \pm 2.07$ & $5.67 \pm 0.82$ & $8.33 \pm 2.33$ \\
\hline $\mathrm{C}_{\max }(\mathrm{ng} / \mathrm{ml})$ & $16.9 \pm 5.1$ & $44.0 \pm 8.3$ & $29.3 \pm 8.1$ & $34.9 \pm 9.8$ & $21.3 \pm 4.4$ \\
\hline Metabolic conversion (\%) & $19.6 \pm 8.1$ & $21.2 \pm 5.99$ & $25.2 \pm 6.6$ & $25.7 \pm 5.0$ & $21.8 \pm 3.3$ \\
\hline \multicolumn{6}{|l|}{$N$-Desmethyltamoxifen } \\
\hline AUC (h ng/ml) & $283.7 \pm 61.8$ & $743.7 \pm 81.9$ & $354.9 \pm 96.4$ & $460.7 \pm 139.5$ & $243.2 \pm 40.7$ \\
\hline$t_{1 / 2}(h)$ & $16.4 \pm 10.4$ & $12.3 \pm 9.2$ & $11.4 \pm 2.0$ & $8.09 \pm 2.11$ & $5.67 \pm 3.40$ \\
\hline $\mathrm{T}_{\max }(\mathrm{h})$ & $9.33 \pm 2.07$ & $8.00 \pm 4.56$ & $10.3 \pm 2.3$ & $8.67 \pm 2.73$ & $9.67 \pm 2.66$ \\
\hline $\mathrm{C}_{\max }(\mathrm{ng} / \mathrm{ml})$ & $11.9 \pm 2.7$ & $33.7 \pm 12.4$ & $17.1 \pm 6.3$ & $23.2 \pm 9.5$ & $15.1 \pm 2.1$ \\
\hline Metabolic conversion (\%) & $16.1 \pm 2.4$ & $22.2 \pm 4.9$ & $17.2 \pm 3.3$ & $22.4 \pm 3.9$ & $15.9 \pm 2.2$ \\
\hline \multicolumn{6}{|l|}{ Endoxifen } \\
\hline AUC (h ng/ml) & $92.7 \pm 44.2$ & $546.9 \pm 203.8^{\star}$ & $454.9 \pm 103.1^{*}$ & $449.1 \pm 127.9^{*}$ & $258.6 \pm 52.8^{\star \# \mathbb{R}}$ \\
\hline$t_{1 / 2}(h)$ & $7.48 \pm 2.57$ & $11.8 \pm 3.5$ & $10.2 \pm 3.9$ & $9.74 \pm 3.98$ & $13.4 \pm 22.7$ \\
\hline $\mathrm{T}_{\max }(\mathrm{h})$ & $8.00 \pm 4.00$ & $11.0 \pm 4.3$ & $9.67 \pm 2.33$ & $12.5 \pm 4.6$ & $12.3 \pm 3.2$ \\
\hline $\mathrm{C}_{\max }(\mathrm{ng} / \mathrm{ml})$ & $4.77 \pm 1.36$ & $21.7 \pm 6.2$ & $20.2 \pm 4.0$ & $19.9 \pm 5.1$ & $13.6 \pm 4.4$ \\
\hline Metabolic conversion (\%) & $4.73 \pm 2.27$ & $16.8 \pm 7.2^{*}$ & $23.0 \pm 8.3^{*}$ & $22.0 \pm 4.4^{*}$ & $17.1 \pm 4.1^{\star}$ \\
\hline
\end{tabular}

$A \cup C_{0-\infty}$ area under the plasma concentration-time curve from $0 \mathrm{~h}$ to infinity; $C_{\max }$ : peak plasma concentration; $t_{\max }$ time to reach $C_{\max ;} t_{1 / 2}:$ terminal half-life. ${ }^{*} p<0.05$, a significant difference compared with the sham group within the group by one-way ANOVA and Student-Newman-Keuls post-hoc tests. ${ }^{p} p<0.05$, a significant difference compared with the concurrent $R T_{2.0 G y}$ group. $\mathbb{P}_{p}<0.05$, a significant difference compared with the sequential $R T_{0.5 G y}$ group. Data are expressed as the mean \pm standard deviation ( $n=6$ per group). 

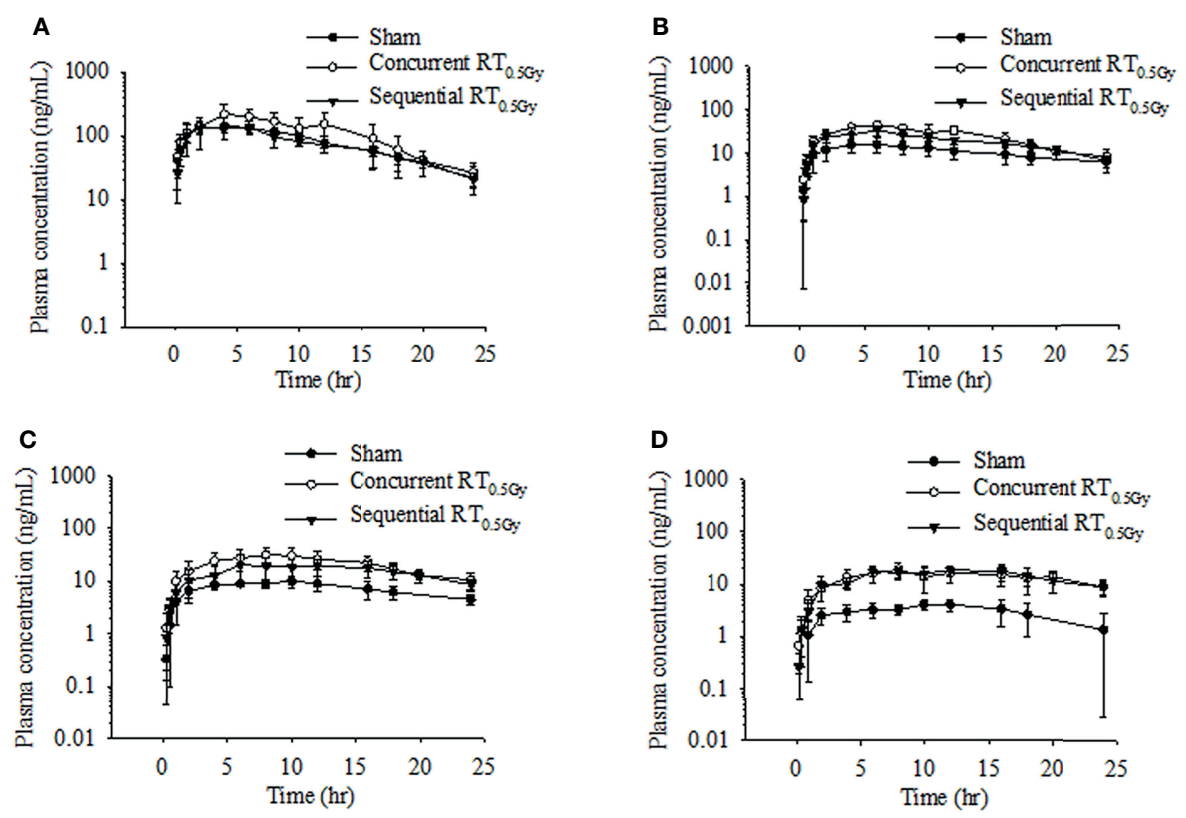

FIGURE 2 | Time-concentration curve of tamoxifen (A), 4-hydroxytamoxifen (B), N-desmethyltamoxifen (C), and endoxifen (D) in rat plasma after oral administration

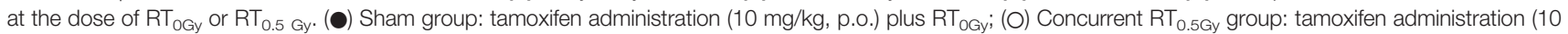

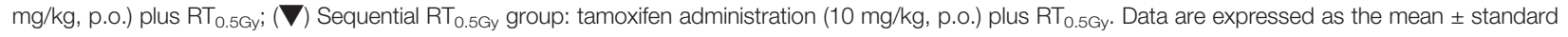
deviation ( $n=6$ per group).
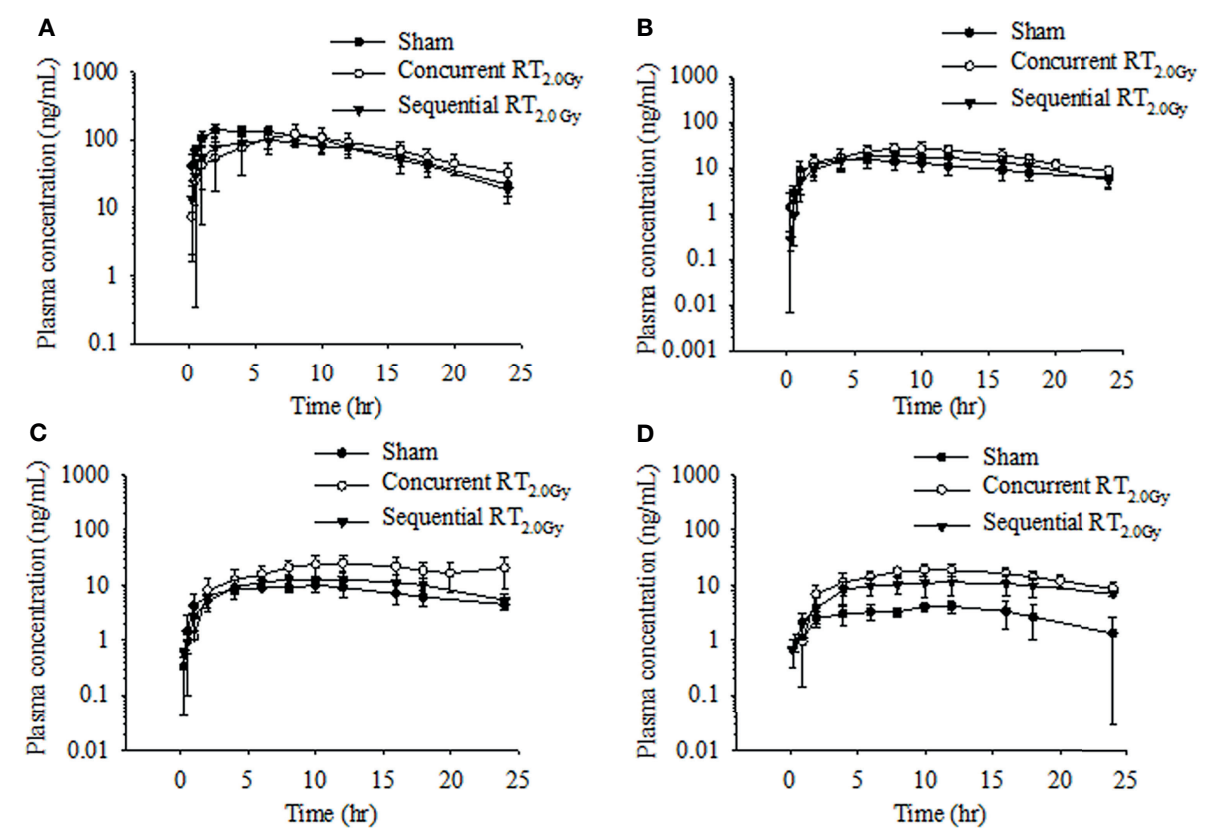

FIGURE 3 | Time-concentration curve of tamoxifen (A), 4-hydroxytamoxifen (B), N-desmethyltamoxifen (C), and endoxifen (D) in rat plasma after oral administration at the dose of $\mathrm{RT}_{\mathrm{OGy}}$ or $\mathrm{RT}_{2.0}$ Gy. (O) Sham group: tamoxifen administration (10 mg/kg, p.o.) plus $\mathrm{RT}_{\mathrm{OGy}}$; (O) Concurrent $\mathrm{RT}_{2.0 \mathrm{~Gy}}$ group: tamoxifen administration (10 $\mathrm{mg} / \mathrm{kg}$, p.o.) plus $\mathrm{RT}_{2.0 \mathrm{gy}}$; $\boldsymbol{\nabla}$ ) Sequential $\mathrm{RT}_{2.0 \mathrm{gy}}$ group: tamoxifen administration (10 mg/kg, p.o.) plus $\mathrm{RT}_{2.0 \mathrm{gy}}$. Data are expressed as the mean \pm standard deviation ( $n=6$ per group). 
of tamoxifen and $\mathrm{N}$-desmethyltamoxifen was 2 -fold higher than the respective $\mathrm{C}_{\max }$ at the sham and $\mathrm{RT}_{2.0 \mathrm{~Gy}}$ groups. The AUC value in the $\mathrm{RT}_{0.5 \mathrm{~Gy}}$ group was 1.5 - to 1.7 -fold higher than in the sham and $\mathrm{RT}_{2.0 \mathrm{~Gy}}$ groups. As shown in Table 1, the relative bioavailability of tamoxifen was higher in $\mathrm{RT}_{0.5 \mathrm{~Gy}}$ (ranging from $127 \%$ to $202 \%$ ) than in $\mathrm{RT}_{2.0 \mathrm{~Gy}}$ (ranging from $71 \%$ to $152 \%$ ). Regarding the relevant $\mathrm{PK}$ parameters, half-lives and $\mathrm{T}_{\max }$ were not significantly different from the sham group. However, compared to the concurrent irradiated groups with the sham group, the metabolic conversion of $\mathrm{N}$-desmethyltamoxifen and endoxifen was significantly increased. The 4-hydroxytamoxifen conversion ratio was not significantly affected by the concurrent regime. Interestingly, however, the magnitude of endoxifen converted from 4-hydroxytamoxifen and $\mathrm{N}$-desmethyltamoxifen increased 3- to 5-fold.

\section{Pharmacokinetics and Biotransformation of Tamoxifen During the Sequential Regimen}

In this condition, tamoxifen was administered $24 \mathrm{~h}$ after irradiation with either 0.5 Gy or 2.0 Gy. The mean plasma concentration-time profile following single oral administration of tamoxifen with $\mathrm{RT}_{0.5 \mathrm{~Gy}}$ and $\mathrm{RT}_{2.0 \mathrm{~Gy}}$ is shown in Figures $\mathbf{2}$ and $\mathbf{3}$. There were no significant differences in AUCs between the sham and sequential $\mathrm{RT}_{0.5 \mathrm{~Gy}}$ groups. Additionally, the metabolic conversion for 4-hydroxytamoxifen and $\mathrm{N}$-desmethyltamoxifen was similar in the sequential $\mathrm{RT}_{0.5 \mathrm{~Gy}}$ and sham groups except for endoxifen. Nevertheless, the AUC of tamoxifen in the sequential $\mathrm{RT}_{2.0 \mathrm{~Gy}}$ group decreased by $24 \%$; the relative biotransformation (\%) was decreased by approximately $76 \%$, compared to the sham group. Regarding metabolic conversion in the $\mathrm{RT}_{2.0 \mathrm{~Gy}}$ treatment, the conversion level of 4-hydroxytamoxifen and $\mathrm{N}$ desmethyltamoxifen was not significantly affected. However, for the $\mathrm{RT}_{2.0 \mathrm{~Gy}}$ group, endoxifen conversion was four times higher than that of the non-irradiated group. There were no other significant effects of RT on the various PK parameters.

The change of tamoxifen metabolites between the concurrent and sequential regimens was analyzed. The respective AUC of 4hydroxytamoxifen increased by $83 \%$ and $37 \%$ in the concurrent $\mathrm{RT}_{0.5 \mathrm{~Gy}}$ and $\mathrm{RT}_{2.0 \mathrm{~Gy}}$ groups, respectively. By $36 \%$ of the increase in the sequential $\mathrm{RT}_{0.5 \mathrm{~Gy}}$ group. In the concurrent $\mathrm{RT}_{0.5 \mathrm{~Gy}}$ and $\mathrm{RT}_{2.0 \mathrm{~Gy}}$ groups, the AUC of endoxifen increased by $488 \%$ and $389 \%$, respectively; the respective metabolic conversion ratio of endoxifen for tamoxifen concurrent with $\mathrm{RT}_{0.5 \mathrm{~Gy}}$ and $\mathrm{RT}_{2.0 \mathrm{~Gy}}$ was 3.4- and 4.6fold higher than the sham group. Furthermore, the AUC of endoxifen in the sequential regimen increased in the $\mathrm{RT}_{0.5 \mathrm{~Gy}}$ group, by $383 \%$ and $178 \%$, respectively. The metabolic conversion rate of endoxifen for tamoxifen sequential with $\mathrm{RT}_{0.5 \mathrm{~Gy}}$ and $\mathrm{RT}_{2.0 \mathrm{~Gy}}$ was 4.4- and 3.4fold that of the sham group, respectively.

\section{DISCUSSION}

The biochemical pathways responsible for tamoxifen metabolism are complex, involving recently discovered estragon-like tamoxifen metabolites and cytochrome $\mathrm{P} 450$-dominated processes, particularly CYP3A4 CYP2D6 isomers (27). In short, tamoxifen is metabolized from 4-hydroxylated to 4-hydroxytamoxifen by CYP2D6 isomers and from $N$-dealkylated to $N$ desmethyltamoxifen by CYP3A4 isomers. $N$-desmethyltamoxifen and 4-hydroxytamoxifen individually undergo these reactions to form the secondary metabolites, $\mathrm{N}, \mathrm{N}$-didesmethyltamoxifen, and endoxifen (27). Among them, 4-hydroxytamoxifen and endoxifen modulate the therapeutic efficacy of tamoxifen due to their competitive binding to estragon receptors with a binding affinity 30-100 times higher than tamoxifen itself, resulting in the inhibition of breast tumor growth $(27,28)$.

According to the Surveillance, Epidemiology, and End Results (SEER) data from 2005 to 2010, the incidence for male breast cancer was 2,054 male/289,673 all breast cancer cases (29). Male breast cancer is almost hormone receptor positive, and the use of endocrine therapy such as tamoxifen is routine for managing male breast cancer (30). In comparing our previous study (24) and the current study, the AUC of tamoxifen in female rats was about 1.2-fold higher than that in male rats. Moreover, the metabolic conversion ratio of 4-hydroxytamoxifen to $\mathrm{N}$ desmethyltamoxifen in female rats was roughly onefold and the ratio of 4-hydroxytamoxifen to endoxifen conversion was 4- to 5fold. However, our previous study demonstrated that the percentage of 4-hydroxytamoxifen conversion in male rats is only $26 \%$ (24). The endoxifen conversion ratio in female rats is significantly greater than that in male rats, implying that the endoxifen conversion ratio is higher in female rats than in male rats.

It has been reported that RT affects the cytochrome P450 enzyme, resulting in the change of drug pharmacokinetics $(16,31)$. Consequently, in the present study, we investigated the RTtamoxifen combination on pharmacokinetic behavior and biotransformation. As Figure 3 illustrated, the $\mathrm{T}_{\max }$ of tamoxifen was 3 to $4 \mathrm{~h}$ more prolonged in the concurrent $\mathrm{RT}_{2.0 \mathrm{~Gy}}$ group compared with the sham group. Conversely, in the sequential $\mathrm{RT}_{2.0 \mathrm{~Gy}}$ group, no significant effects were observed. As previously discussed, endoxifen and 4-hydroxytamoxifen have an approximately 100-fold greater affinity for the estrogen receptor (ER) and the ability to inhibit cell proliferation. Additionally, 4-hydroxytamoxifen has been shown to cause specific ER-mediated and non-specific ER-independent cytotoxic effects in a dose-dependent manner (32). Moreover, endoxifen exhibits superior antiestrogenic activity than the parent drug (33), and the concentration of endoxifen is probably responsible for the clinical outcomes of breast cancer patients receiving adjuvant tamoxifen treatment (34).

Recent NSABP-B14 and NSABP B-24 trials reported that concurrent tamoxifen with RT has a higher probability of local control for patients with breast cancer $(9,35)$. However, sequential tamoxifen treatment following RT is more effective than concurrent treatment (36). Moreover, the addition of RT to adjuvant tamoxifen reduces the number of in-breast recurrences for older women from 80 to 20 (95\% CI $10-40)$ per 1,000 patients at 10 years (37). Interestingly, in a recent prospective randomized study comparing breast pain after breast-conserving surgery plus tamoxifen with or without RT (38), the incidence and severity of breast symptoms were similar at baseline in patients subsequently randomized to the RT and no-RT arms. Nevertheless, Azria D and colleagues reported 
that concomitant use of tamoxifen with RT increases the incidence of grade 2 subcutaneous fibrosis in hypersensitive patients (39). Also, the risk of pulmonary fibrosis is increased by $20 \%$ for breast cancer patients treated with concurrent regimens $(11,12)$.

According to our findings, the AUC of endoxifen was significantly enhanced in the $\mathrm{RT}_{2.0 \mathrm{~Gy}}$ and $\mathrm{RT}_{0.5 \mathrm{~Gy}}$ groups. Endoxifen is converted from $\mathrm{N}$-desmethyltamoxifen by the critical phase I enzyme, CYP2D6, responsible for the endoxifen level (40). Moreover, previous evidence has shown that poor and ultrarapid CYP2D6 metabolizers of tamoxifen could predict worse clinical outcomes among patients with breast cancer treated with tamoxifen (41). The association between CYP2D6 ${ }^{*} 4$ and radiation toxicity has been reported by Damaraju and coworkers (42). However, CYP2D6 ${ }^{\star} 4$ is one of the typical CYP2D6 inactive alleles with nonfunctional CYP2D6 activity (27). Our pharmacokinetic profiles and results implied that the 4-hydroxytamoxifen, $\mathrm{N}$ desmethyltamoxifen, and endoxifen levels are enhanced in both the irradiated 2.0-Gy groups. In the $\mathrm{RT}_{2.0 \mathrm{~Gy}}$ groups, the increasing magnitude of the sequential regimen is slightly lower than the concurrent regimen. Significantly, the endoxifen level and conversion ratio after RT were increased compared with the sham group. Based on these findings, local chest wall irradiation with both 0.5 and 2 Gy probably influences the cytochrome P450 family activity, resulting in altered drug concentration in plasma and drug biotransformation. Additionally, the similar trend of endoxifen converted by tamoxifen in the concurrent and sequential regimens with RT 0.5 and 2 Gy may partly explain why both regimens provide effective clinical outcomes $(9,35,36)$.

Interestingly, the AUC levels of tamoxifen and metabolites in the sequential regimen were opposite for the $\mathrm{RT}_{2.0 \mathrm{~Gy}}$ and $\mathrm{RT}_{0.5 \mathrm{~Gy}}$ groups. Compared with the sequential group, the AUC for tamoxifen in the sham group increased by $1 \%$ in the $\mathrm{RT}_{0.5 \mathrm{~Gy}}$ group but decreased by $24 \%$ in the $\mathrm{RT}_{2.0 \mathrm{~Gy}}(p<0.05)$. These data imply that the tamoxifen may be similarly affected by off-target irradiation as well as nonirradiated group during the sequential regimen and may be exposed under a tamoxifen level of similar magnitude to that of the nonirradiated group. On the contrary, as Figure 2 shows, in the concurrent $\mathrm{RT}_{0.5 \mathrm{~Gy}}$ group, the AUC levels of tamoxifen are appreciably more significant than that in the concurrent $\mathrm{RT}_{2.0 \mathrm{~Gy}}$ and sham groups. However, there were no differences in AUC levels between the concurrent $\mathrm{RT}_{2.0 \mathrm{~Gy}}$ and sham groups. Higher concentrations of tamoxifen or toxic metabolites may lead to adverse effects or better control (28). Therefore, the relatively higher tamoxifen level in the concurrent $\mathrm{RT}_{0.5 \mathrm{~Gy}}$ group may be considered a consequence of irradiation to the off-target area.

Recently, it was found that protein expression and activity could be changed by doses of RT dose as low as 1.0 Gy (43). RT may impair the vascular and lymphatic systems, thus causing endothelial cell loss (44), which has been associated with enhanced vascular permeability that may enhance the drug's easier permeation into the lesion from blood circulation and can effectively reach the target despite lower tamoxifen levels. In the current study, tamoxifen translated to endoxifen was increased even after being irradiated 0.5 Gy. The findings imply that the irradiated surrounding normal tissues tolerated the high tamoxifen level during the concurrent regimen. However, tamoxifen has a narrow therapeutic window.
Either the extreme change of drug level or the high-dose exposure under the narrow-therapeutic index drugs probably enhances the cytotoxicity to the surrounding normal tissues. These lines of PK's data support the conversional effect of tamoxifen and could be modulated by RT in both concurrent and sequential regimens. More importantly, the current data also point out the potential toxicity caused by off-target doses for patients with breast cancer treated with advanced RT techniques and tamoxifen.

Most breast cancer tumors are generally considered immunologically "cold", with low immune cell infiltration, and are highly difficult to target with immunotherapy (45). Recently, Wolfson and colleagues (46) demonstrated that tamoxifen and 4hydroxytamoxifen sensitize breast cancer cells to natural killer (NK) cell-mediated killing as immunomodulatory agents regardless of estrogen receptor expression. Additionally, G protein-coupled receptor 30 (GPR30, also known as G proteincoupled estrogen receptor, GPER) binds estrogen (47) and as ligands for tamoxifen (48) and 4-hydroxytamoxifen (49). Moreover, GPR30 has also been shown to be associated with extracellular signal-regulated kinase-mediated phosphatidylinositol 3-kinase (PI3K)/serine/threonine kinase Akt signaling pathways (50). Moreover, RT induced ROS generation and nuclear factor$\mathrm{\kappa B}$ activation, and facilitated polymorphonuclear cell leukocyte accumulation (51) that influences the immune response (52). Intriguingly, RT activates mitogen-activated protein kinase and PI3K pathway (53). These lines of evidence support a rationale for further investigation of combination with ER targeting drugs, immuno-oncology agents, and RT.

There were some limitations to our study. First, the current study was designed to examine the interaction between RT and the PK of tamoxifen, but it does not include the pharmacodynamics of tamoxifen during RT. However, the published clinical data support the synergistic effect of treatment and toxicity. Additionally, the current analysis sheds light on the discrepancies of $\mathrm{PK}$ in the concurrent and sequential regimens of RT with tamoxifen, which will be helpful in the clinical setting. Second, the current study used a single fraction instead of multiple daily fractions to investigate the interaction between RT and tamoxifen. Third, the current study used normal and healthy rats instead of rats with breast cancer disease model. However, the present findings addressed the basic pharmacokinetic interaction between radiation and tamoxifen that established the foundation of the relevant disease models. Although these limitations are present, our previous study suggested that the AUC of drugs could be influenced by multiple fractions and a single fraction (31). Hence, the interaction of tamoxifen between multiple fractions model is warranted in the future. The possible mechanism was not examined in the current study because the presence or absence of the RT-PK phenomenon in the context of tamoxifen plus RT could not be ensured before the study. After the study, the RT-PK phenomenon of tamoxifen is confirmed, and the possible mechanism of the disease model should be examined in the future.

To our best knowledge, the current study is the first to confirm the RT-drug interaction with RT-PK phenomenon between RT, tamoxifen, and metabolites. RT could modulate the systemic PK of tamoxifen and metabolites with off-target and 
treatment doses with different combinations of regimens. These findings provide the rationale for further studies to investigate the interactive effects of RT on the pharmacokinetics and biotransformation of tamoxifen.

\section{DATA AVAILABILITY STATEMENT}

The original contributions presented in the study are included in the article/supplementary material. Further inquiries can be directed to the corresponding authors.

\section{ETHICS STATEMENT}

The animal study was reviewed and approved by IUCAC no. 1051204. Written informed consent was obtained from the owners for the participation of their animals in this study.

\section{REFERENCES}

1. Sung H, Ferlay J, Siegel RL, Laversanne M, Soerjomataram I, Jemal A, et al. Global Cancer Statistics 2020: GLOBOCAN Estimates of Incidence and Mortality Worldwide for 36 Cancers in 185 Countries. CA Cancer J Clin (2021) 71:209-49. doi: 10.3322/caac.21660

2. Smith IE, Ross GM. Breast Radiotherapy After Lumpectomy-No Longer Always Necessary. N Engl J Med (2004) 351:1021-3. doi: 10.1056/NEJMe048173

3. Poortmans PM, Collette S, Kirkove C, Van Limbergen E, Budach V, Struikmans $\mathrm{H}$, et al. Internal Mammary and Medial Supraclavicular Irradiation in Breast Cancer. N Engl J Med (2015) 373:317-27. doi: 10.1056/NEJMoa1415369

4. Poortmans PM, Struikmans H, Bartelink H. Regional Nodal Irradiation in Early-Stage Breast Cancer. N Engl J Med (2015) 373:1879-80. doi: 10.1056/ NEJMc1510505

5. Cuzick J, Powles T, Veronesi U, Forbes J, Edwards R, Ashley S, et al. Overview of the Main Outcomes in Breast-Cancer Prevention Trials. Lancet (2003) 361:296-300. doi: 10.1016/s0140-6736(03)12342-2

6. Veronesi U, Maisonneuve P, Costa A, Sacchini V, Maltoni C, Robertson C, et al. Prevention of Breast Cancer With Tamoxifen: Preliminary Findings From the Italian Randomised Trial Among Hysterectomised Women. Italian Tamoxifen Prevention Study. Lancet (1998) 352:93-7. doi: 10.1016/s01406736(98)85011-3

7. Fisher B, Costantino JP, Wickerham DL, Redmond CK, Kavanah M, Cronin WM, et al. Tamoxifen for Prevention of Breast Cancer: Report of the National Surgical Adjuvant Breast and Bowel Project P-1 Study. J Natl Cancer Inst (1998) 90:1371-88. doi: 10.1093/jnci/90.18.1371

8. Hata Y, Takahashi H, Todo S, Okazaki M, Asaishi K, Hirata K, et al. Ten-Year Results of a Randomized Trial on Adjuvant Chemo-Endocrine Therapy With Tamoxifen for Stage II Breast Cancer. Breast Cancer (2003) 10:134-9. doi: $10.1007 / \mathrm{BF} 02967638$

9. Fisher B, Costantino J, Redmond C, Poisson R, Bowman D, Couture J, et al. A Randomized Clinical Trial Evaluating Tamoxifen in the Treatment of Patients With Node-Negative Breast Cancer Who Have Estrogen-Receptor-Positive Tumors. NEngl J Med (1989) 320:479-84. doi: 10.1056/nejm198902233200802

10. Ellis PA, Saccani-Jotti G, Clarke R, Johnston SR, Anderson E, Howell A, et al. Induction of Apoptosis by Tamoxifen and ICI 182780 in Primary Breast Cancer. Int J Cancer (1997) 72:608-13. doi: 10.1002/(sici)1097-0215 (19970807)72:4<608::aid-ijc10>3.0.co;2-7

11. Bentzen SM, Skoczylas JZ, Overgaard M, Overgaard J. Radiotherapy-Related Lung Fibrosis Enhanced by Tamoxifen. J Natl Cancer Inst (1996) 88:918-22. doi: $10.1093 /$ jnci/88.13.918

\section{AUTHOR CONTRIBUTIONS}

Designed and executed the research, Y-YC. Prepared the original draft, Y-YC, TZ, MC, and C-HH. Analyzed the research data, TZ, MC, and Y-YC. Editing the manuscript, Y-YC, C-HH, JD, and T-HT. Supervision, project administration, and funding acquisition, C-HH, Y-JC, and T-HT. All authors contributed to the article and approved the submitted version.

\section{FUNDING}

This work was supported in part by grants from Far Eastern Memorial Hospital (FEMH 110-2314-B-418-006), the Ministry of Science and Technology, Taiwan (MOST 110-2314-B-418006; MOST 110-2981-I-239-001; MOST 110-2113-M-A49A503; MOST 109-2314-B-195-003-MY3), the MacKay Memorial Hospital (MMH-E-110-13; MMH-E-111-11), and the NYMUFEMH Joint Research Program (110DN38).

12. Koc M, Polat P, Suma S. Effects of Tamoxifen on Pulmonary Fibrosis After Cobalt-60 Radiotherapy in Breast Cancer Patients. Radiother Oncol (2002) 64:171-5. doi: 10.1016/s0167-8140(02)00136-6

13. Whelan T, Levine M. Radiation Therapy and Tamoxifen: Concurrent or Sequential? That Is the Question. J Clin Oncol (2005) 23:1-4. doi: 10.1200/ jco.2005.08.955

14. Ogawa Y, Kobayashi T, Nishioka A, Kariya S, Hamasato S, Seguchi H, et al. Radiation-Induced Reactive Oxygen Species Formation Prior to Oxidative DNA Damage in Human Peripheral T Cells. Int J Mol Med (2003) 11:149-52. doi: 10.3892/ijmm.11.2.149

15. Loguercio C, Federico A. Oxidative Stress in Viral and Alcoholic Hepatitis Free Radic Biol Med (2003) 34:1-10. doi: 10.1016/s0891-5849(02)01167-x

16. Hsieh C-H, Chen Y-J, Tsai T-H, Wang L-Y, Tai H-C, Huang H-L, et al. Robust Combination of Liver Stereotactic Body Radiotherapy Modulates Pharmacokinetics of Sorafenib Toward Preferable Parameters. Sci Rep (2020) 10:9575-88. doi: 10.1038/s41598-020-66583-9

17. Tsai TH, Chen YJ, Wang LY, Hsieh CH. Impact of Local Liver Irradiation Concurrent Versus Sequential With Lenvatinib on Pharmacokinetics and Biodistribution. Cancers (2021) 13:1598-612. doi: 10.3390/cancers13071598

18. Yeh HP, Huang YC, Wang LY, Shueng PW, Tien HJ, Chang CH, et al. Helical Tomotherapy With a Complete-Directional-Complete Block Technique Effectively Reduces Cardiac and Lung Dose for Left-Sided Breast Cancer. $\mathrm{Br}$ J Radiol (2020) 93:20190792. doi: 10.1259/bjr.20190792

19. Darby SC, Ewertz M, McGale P, Bennet AM, Blom-Goldman U, Brønnum D, et al. Risk of Ischemic Heart Disease in Women After Radiotherapy for Breast Cancer. N Engl J Med (2013) 368:987-98. doi: 10.1056/nejmoa1209825

20. Bratherton DG, Brown CH, Buchanan R, Hall V, Kingsley Pillers EM, Wheeler TK, et al. A Comparison of Two Doses of Tamoxifen (Nolvadex) in Postmenopausal Women With Advanced Breast Cancer: $10 \mathrm{Mg}$ Bd Versus 20 Mg Bd. Br J Cancer (1984) 50:199-205. doi: 10.1038/bjc.1984.163

21. Kiyotani K, Mushiroda T, Imamura CK, Tanigawara Y, Hosono N, Kubo M, et al. Dose-Adjustment Study of Tamoxifen Based on CYP2D6 Genotypes in Japanese Breast Cancer Patients. Breast Cancer Res Treat (2012) 131:137-45. doi: 10.1007/s10549-011-1777-7

22. Reagan-Shaw S, Nihal M, Ahmad N. Dose Translation From Animal to Human Studies Revisited. FASEB J (2008) 22:659-61. doi: 10.1096/fj.079574LSF

23. Kim CS, Choi SJ, Park CY, Li C, Choi JS. Effects of Silybinin on the Pharmacokinetics of Tamoxifen and Its Active Metabolite, 4Hydroxytamoxifen in Rats. Anticancer Res (2010) 30:79-85.

24. Cheng Y-Y, Tuzo ET, Dalley JW, Tsai T-H. Dose-Dependent Effects of Hedyotis Diffusa Extract on the Pharmacokinetics of Tamoxifen, 4- 
Hydroxytamoxifen, and N-Desmethyltamoxifen. Biomed Pharmacother (2022) 145:112466. doi: 10.1016/j.biopha.2021.112466

25. Hsieh CH, Hsieh YJ, Liu CY, Tai HC, Huang YC, Shueng PW, et al. Abdominal Irradiation Modulates 5-Fluorouracil Pharmacokinetics. J Transl Med (2010) 8:29. doi: 10.1186/1479-5876-8-29

26. Vriesendorp HM, Van Bekkum DW. Susceptibility to Total-Body Irradiaiton. In: Broerse JJ, MacVittle TJ, editors. Springer, Dordrecht. Response to TotalBody Irradiation in Different Species Boston: Martinus Nijhoff (1984). p. 43-57. doi: 10.1007/978-94-009-6048-0-4

27. Sanchez-Spitman AB, Swen JJ, Dezentje VO, Moes D, Gelderblom H, Guchelaar HJ. Clinical Pharmacokinetics and Pharmacogenetics of Tamoxifen and Endoxifen. Expert Rev Clin Pharmacol (2019) 12:523-36. doi: 10.1080/17512433.2019.1610390

28. Dezentje VO, Guchelaar HJ, Nortier JW, van de Velde CJ, Gelderblom H. Clinical Implications of CYP2D6 Genotyping in Tamoxifen Treatment for Breast Cancer. Clin Cancer Res (2009) 15:15-21. doi: 10.1158/1078-0432.CCR-08-2006

29. Liu N, Johnson KJ, Ma CX. Male Breast Cancer: An Updated Surveillance, Epidemiology, and End Results Data Analysis. Clin Breast Cancer (2018) 18: e997-e1002. doi: 10.1016/j.clbc.2018.06.013

30. Gucalp A, Traina TA, Eisner JR, Parker JS, Selitsky SR, Park BH, et al. Male Breast Cancer: A Disease Distinct From Female Breast Cancer. Breast Cancer Res Treat (2019) 173:37-48. doi: 10.1007/s10549-018-4921-9

31. Tsai TH, Chen YJ, Wang LY, Hsieh CH. Effect of Synchronous Versus Sequential Regimens on the Pharmacokinetics and Biodistribution of Regorafenib with Irradiation. Pharmaceutics (2021) 13:386-404. doi: 10.3390/pharmaceutics13030386

32. Bardon S, Vignon F, Montcourrier P, Rochefort H. Steroid Receptor-Mediated Cytotoxicity of an Antiestrogen and an Antiprogestin in Breast Cancer Cells. Cancer Res (1987) 47:1441-8.

33. Wu X, Hawse JR, Subramaniam M, Goetz MP, Ingle JN, Spelsberg TC. The Tamoxifen Metabolite, Endoxifen, Is a Potent Antiestrogen That Targets Estrogen Receptor Alpha for Degradation in Breast Cancer Cells. Cancer Res (2009) 69:1722-7. doi: 10.1158/0008-5472.can-08-3933

34. Sanchez-Spitman AB, Moes DAR, Swen JJ, Dezentjé VO, Lambrechts D, Neven P, et al. Exposure-Response Analysis of Endoxifen Serum Concentrations in Early-Breast Cancer. Cancer Chemother Pharmacol (2020) 85:1141-52. doi: 10.1007/s00280-020-04089-x

35. Fisher B, Dignam J, Wolmark N, Wickerham DL, Fisher ER, Mamounas E, et al. Tamoxifen in Treatment of Intraductal Breast Cancer: National Surgical Adjuvant Breast and Bowel Project B-24 Randomised Controlled Trial. Lancet (1999) 353:1993-2000. doi: 10.1016/s0140-6736(99)05036-9

36. Lenehan JG, Teft WA, Kim RB. Comparison of Endoxifen Levels Between Male and Female Breast Cancer Patients Treated With Tamoxifen. J Clin Oncol (2016) 34:578-8. doi: 10.1200/JCO.2016.34.15_suppl.578

37. Chesney TR, Yin JX, Rajaee N, Tricco AC, Fyles AW, Acuna SA, et al. Tamoxifen With Radiotherapy Compared With Tamoxifen Alone in Elderly Women With Early-Stage Breast Cancer Treated With Breast Conserving Surgery: A Systematic Review and Meta-Analysis. Radiother Oncol (2017) 123:1-9. doi: 10.1016/j.radonc.2017.02.019

38. Wennberg B, Gagliardi G, Sundbom L, Svane G, Lind P. Early Response of Lung in Breast Cancer Irradiation: Radiologic Density Changes Measured by CT and Symptomatic Radiation Pneumonitis. Int J Radiat Oncol Biol Phys (2002) 52:1196-206. doi: 10.1016/s0360-3016(01)02770-5

39. Azria D, Gourgou S, Sozzi WJ, Zouhair A, Mirimanoff RO, Kramar A, et al. Concomitant Use of Tamoxifen With Radiotherapy Enhances Subcutaneous Breast Fibrosis in Hypersensitive Patients. Br J Cancer (2004) 91:1251-60. doi: 10.1038/sj.bjc.6602146

40. Abraham JE, Maranian MJ, Driver KE, Platte R, Kalmyrzaev B, Baynes C, et al. CYP2D6 Gene Variants: Association With Breast Cancer Specific Survival in a Cohort of Breast Cancer Patients From the United Kingdom Treated With Adjuvant Tamoxifen. Breast Cancer Res (2010) 12:R64. doi: 10.1186/bcr2629

41. He W, Grassmann F, Eriksson M, Eliasson E, Margolin S, Thorén L, et al. CYP2D6 Genotype Predicts Tamoxifen Discontinuation and Prognosis in Patients With Breast Cancer. J Clin Oncol (2020) 38:548-57. doi: 10.1200/jco.19.01535

42. Damaraju S, Murray D, Dufour J, Carandang D, Myrehaug S, Fallone G, et al. Association of DNA Repair and Steroid Metabolism Gene Polymorphisms With Clinical Late Toxicity in Patients Treated With Conformal Radiotherapy for Prostate Cancer. Clin Cancer Res (2006) 12:2545-54. doi: 10.1158/10780432.ccr-05-2703

43. Tapio S. Ionizing Radiation Effects on Cells, Organelles and Tissues on Proteome Level. Adv Exp Med Biol (2013) 990:37-48. doi: 10.1007/978-94007-5896-4_2

44. Heisel MA, Laug WE, Stowe SM, Jones PA. Effects of X-Irradiation on Artificial Blood Vessel Wall Degradation by Invasive Tumor Cells. Cancer Res (1984) 44:2441-5.

45. Gatti-Mays ME, Balko JM, Gameiro SR, Bear HD, Prabhakaran S, Fukui J, et al. If We Build It They Will Come: Targeting the Immune Response to Breast Cancer. NPJ Breast Cancer (2019) 5:37-49. doi: 10.1038/s41523-0190133-7

46. Wolfson B, Padget MR, Schlom J, Hodge JW. Exploiting Off-Target Effects of Estrogen Deprivation to Sensitize Estrogen Receptor Negative Breast Cancer to Immune Killing. J Immunother Cancer (2021) 9:e002258. doi: 10.1136/jitc2020-002258

47. Revankar CM, Cimino DF, Sklar LA, Arterburn JB, Prossnitz ER. A Transmembrane Intracellular Estrogen Receptor Mediates Rapid Cell Signaling. Science (2005) 307:1625-30. doi: 10.1126/science.1106943

48. Catalano S, Giordano C, Panza S, Chemi F, Bonofiglio D, Lanzino M, et al. Tamoxifen Through GPER Upregulates Aromatase Expression: A Novel Mechanism Sustaining Tamoxifen-Resistant Breast Cancer Cell Growth. Breast Cancer Res Treat (2014) 146:273-85. doi: 10.1007/s10549-014-3017-4

49. Vivacqua A, Bonofiglio D, Recchia AG, Musti AM, Picard D, Andò S, et al. The G Protein-Coupled Receptor GPR30 Mediates the Proliferative Effects Induced by 17beta-Estradiol and Hydroxytamoxifen in Endometrial Cancer Cells. Mol Endocrinol (2006) 20:631-46. doi: 10.1210/me.2005-0280

50. Filardo EJ, Quinn JA, Bland KI, Frackelton AR Jr. Estrogen-Induced Activation of Erk-1 and Erk-2 Requires the G Protein-Coupled Receptor Homolog, GPR30, and Occurs via Trans-Activation of the Epidermal Growth Factor Receptor Through Release of HB-EGF. Mol Endocrinol (2000) 14:1649-60. doi: 10.1210/mend.14.10.0532

51. Yang L, Kowalski JR, Yacono P, Bajmoczi M, Shaw SK, Froio RM, et al. Endothelial Cell Cortactin Coordinates Intercellular Adhesion Molecule-1 Clustering and Actin Cytoskeleton Remodeling During Polymorphonuclear Leukocyte Adhesion and Transmigration. J Immunol (2006) 177:6440-9. doi: 10.4049/jimmunol.177.9.6440

52. Väyrynen JP, Vornanen J, Tervahartiala T, Sorsa T, Bloigu R, Salo T, et al. Serum MMP-8 Levels Increase in Colorectal Cancer and Correlate With Disease Course and Inflammatory Properties of Primary Tumors. Int J Cancer (2012) 131:E463-74. doi: 10.1002/ijc.26435

53. Cheng JC, Chou CH, Kuo ML, Hsieh CY. Radiation-Enhanced Hepatocellular Carcinoma Cell Invasion With MMP-9 Expression Through PI3K/Akt/NFKappaB Signal Transduction Pathway. Oncogene (2006) 25:7009-18. doi: 10.1038/sj.onc.1209706

Conflict of Interest: The authors declare that the research was conducted in the absence of any commercial or financial relationships that could be construed as a potential conflict of interest.

The reviewer YCH declared a shared affiliation to the with one of the authors, YJC, to the handling editor at time of review.

Publisher's Note: All claims expressed in this article are solely those of the authors and do not necessarily represent those of their affiliated organizations, or those of the publisher, the editors and the reviewers. Any product that may be evaluated in this article, or claim that may be made by its manufacturer, is not guaranteed or endorsed by the publisher.

Copyright (c) 2022 Cheng, Zheng, Chang, Dalley, Chen, Tsai and Hsieh. This is an open-access article distributed under the terms of the Creative Commons Attribution License (CC BY). The use, distribution or reproduction in other forums is permitted, provided the original author(s) and the copyright owner(s) are credited and that the original publication in this journal is cited, in accordance with accepted academic practice. No use, distribution or reproduction is permitted which does not comply with these terms. 